\title{
Erratum to: Induction of initial steps of angiogenic differentiation and maturation of endothelial cells by pericytes in vitro and the role of collagen IV
}

\author{
Zhigang Zhou ${ }^{1,2,3} \cdot$ Friederike Pausch $^{4} \cdot$ Ursula Schlötzer-Schrehardt $^{5}$. \\ Bent Brachvogel $^{6} \cdot$ Ernst Pöschl $^{1}$
}

Published online: 2 April 2016

(C) Springer-Verlag Berlin Heidelberg 2016

\section{Erratum to: Histochem Cell Biol DOI 10.1007/s00418-015-1398-z}

Unfortunately, Figs. 5 and 6 are interchanged in the available online version. Therefore, the figure linked to legend 'Fig. 6' represents Fig. 5 and is described in the text and legend as 'Fig. 5'. Vice versa, the figure linked to legend 'Fig. 5' represents Fig. 6 and is described in the text and legend as 'Fig. 6'.
The legend for Fig. 6 reads as: "Bar $100 \mu \mathrm{m} . \mathrm{d}$ An individual PVC is recruited to the endothelial tube in a region of extended collagen IV deposits". The correct text is: "An individual PVC is recruited to the endothelial tube in a region of extended collagen IV deposits".

We apologize for these errors.

The corrected Figs. 5 and 6 and their legends are given below.

The online version of the original article can be found under doi:10.1007/s00418-015-1398-z.

Ernst Pöschl

e.poschl@uea.ac.uk

1 Biomedical Research Centre, School of Biological Sciences, University of East Anglia, Norwich Research Park, Norwich NR4 7TJ, UK

2 Medical School, University of East Anglia, Norwich Research Park, Norwich, UK

3 Department of Cardiovascular Medicine, Medical College, Nantong University, Nantong, China

4 Department of Experimental Medicine I, University of Erlangen-Nürnberg, Erlangen, Germany

5 Department of Ophthalmology, University ErlangenNürnberg, Erlangen, Germany

6 Medical Faculty, Center for Biochemistry, University of Cologne, Cologne, Germany 
Fig. 5 Pericyte-like vascular cells attach and proliferate differently on various ECM proteins, and integrin $\alpha 1 \beta 1$ mediates adhesion to collagen IV. a, b Attachment of PVC and HUVEC on surfaces coated with collagen I (Coll, $1.25-80 \mu \mathrm{g} / \mathrm{ml})$ and IV (Col4, $0.15-10 \mu \mathrm{g} / \mathrm{ml})$, fibronectin (FN, 0.15-10 $\mu \mathrm{g} / \mathrm{ml}$ ) and laminin 1 (LN-1, 1.25-80 $\mu \mathrm{g} /$ $\mathrm{ml})$. c, d Adhesion of PVC and HUVEC to collagen IV (Col4; black) can be specifically blocked by neutralizing antibodies for integrin $\alpha 1$ (anti- $\alpha 1$ ), but not by anti- $\alpha 2$ or the IgG isotype control. Adhesion to fibronectin (FN; white) is not affected by any neutralizing antibody. Data are expressed as mean $\pm \mathrm{SD}, n=5$
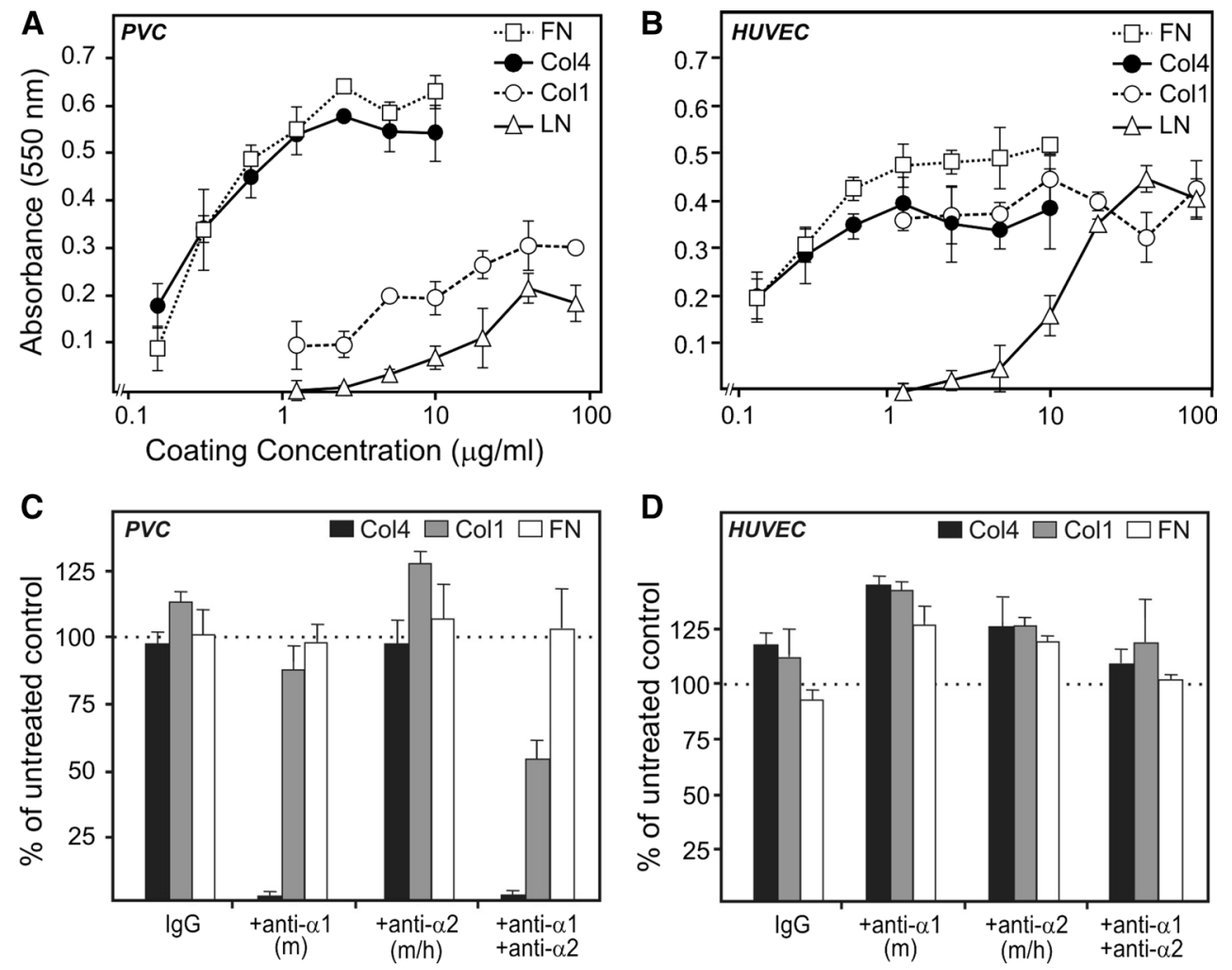
A

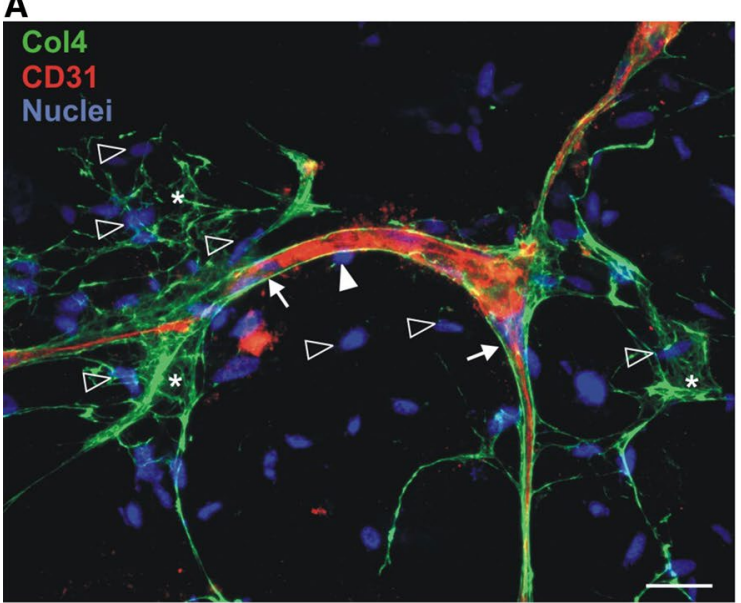

C
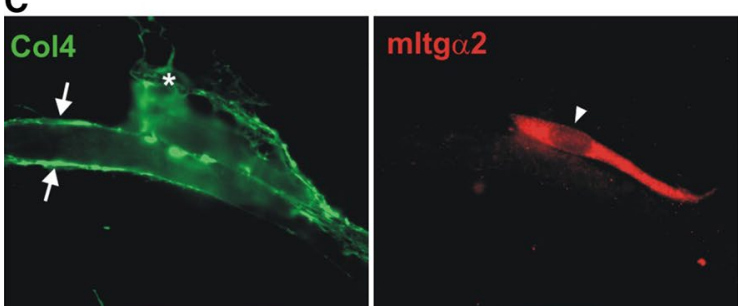

B
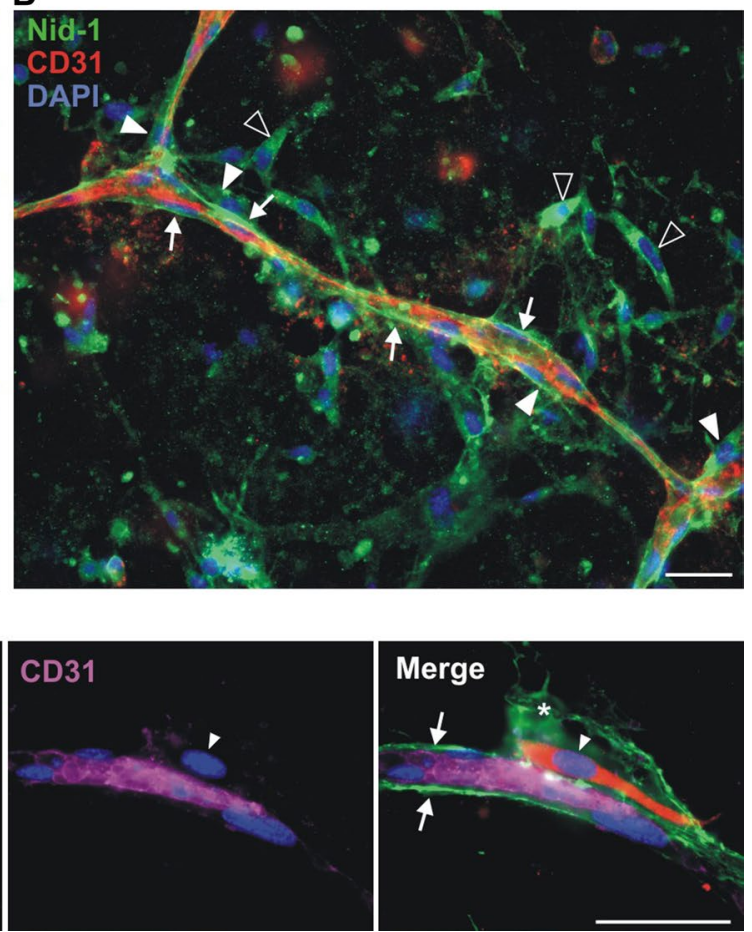

Fig. 6 Collagen IV is expressed by endothelial cells as extended networks, which contribute to PVC recruitment. Cocultures of HUVEC and PVC were immunostained for (a) collagen type IV (Col4, green) or b nidogen-1 (Nid1, green) in combination with CD31 (red), respectively. Tube-associated continuous matrix layers (open arrowhead) as well as an extended collagen IV mesh (filled arrowhead) can be seen, which is also decorated by nidogen-1. PVC (arrows) are indicated by the nuclear stain (blue) but do not express collagen IV. Bar $50 \mu \mathrm{m}$. c The mouse integrin $\alpha 2$ subunit (mIntegrin $\alpha 2$, red) is detected in PVC, which interact with forming tubes marked by collagen IV (Col4, green) and CD31 (magenta). An individual PVC is recruited to the endothelial tube in a region of extended collagen IV deposits. Bar $50 \mu \mathrm{m}$. Merged pictures include nuclear staining (blue) 